\title{
Alkohol kurbelt diabetische Ketoazidose an
}

\author{
Unter höherem Alkoholkonsum leidet die Stoffwechseleinstellung junger Typ-1-Diabetiker. \\ Eine Studie zeigt, dass insbesondere die Rate an diabetischen Ketoazidosen steigt.
}

_ Für 29.630 Jugendliche und junge Erwachsene im Alter von 12-30 Jahren liegen in der DPV-Datenbank Angaben zum durchschnittlichen Alkoholkonsum vor. Diese Patienten wurden entsprechend deutschen Leitlinien in Abstinenzler, Niedrigrisikotrinker (Frauen 0,1-12 g/d, Männer 0,1-24 g/d) und Risikotrinker (Frauen $>12 \mathrm{~g} / \mathrm{d}$, Männer $>24 \mathrm{~g} / \mathrm{d}$ ) kategorisiert. Sodann wurde der Zusammenhang mit verschiedenen Werten aus der Diabetes-Datenbank berechnet.

10,8\% der Patienten gaben Alkoholkonsum an (2,8\% der 12-16 Jährigen, $19,3 \%$ der 18 - bis 25-Jährigen). Ein Risikokonsum lag bei $<0,1 \%$ der 12 - bis
16-Jährigen, $0,3 \%$ der 16 - bis 18 -Jährigen, $1,0 \%$ der 18 - bis 25 -Jährigen und $22 \%$ der 25- bis 30-Jährigen vor.

Das höchste Risiko für schwere Hypoglykämien hatten die Risikotrinker (23,2/100 Patientenjahre, gegenüber 17,0 bei Abstinenzlern; $\mathrm{p}<0,01)$. Nicht signifikant war der Unterschied bei Hypoglykämien mit Bewusstlosigkeit oder Koma (5,0 vs. 4,2/100 Patientenjahre). Der $\mathrm{HbA}_{1 \mathrm{c}}$-Wert lag bei Risikotrinkern im Schnitt bei 9,3\%, bei Niedrigrisikotrinkern bei $8,6 \%$ und bei Nichttrinkern bei $8,4 \%$ ( $\mathrm{p}<0,001)$. Interessanterweise war auch die diabetische Ketoazidose (DKA) mit zunehmendem Alkoholkonsum signifikant wahrscheinlicher: $6,4 \%$ bei
Abstinenz, 7,5\% bei niedrigem und $18,9 \%$ bei hohem Konsum ( $\mathrm{p}<0,001)$.

- Hermann JM et al. Self-reported regular alcohol consumption in adolescents and emerging adults with type 1 diabetes: A neglected risk factor for diabetic ketoacidosis? Multicenter analysis of 29630 patients from the DPV registry. Pediatr Diabetes 2017, online 1. Februar; doi: 10.1111/pedi.12496

\section{KOMMENTAR}

Die Studie zeigt eindrucksvoll die Assoziation zwischen Alkoholkonsum und Stoffwechsel bei Typ-1-Diabetes. Dabei ist die Verbindung zur DKA aus meiner Sicht besonders wichtig. Unsere Schulungen zielen ja aktuell v. a. auf Hypoglykämievermeidung. Sie sollten dringend angepasst werden.

PD Dr. med. T. M. Kapellen

\section{Eosinophile Mittelohrentzündung mit Polypenbildung}
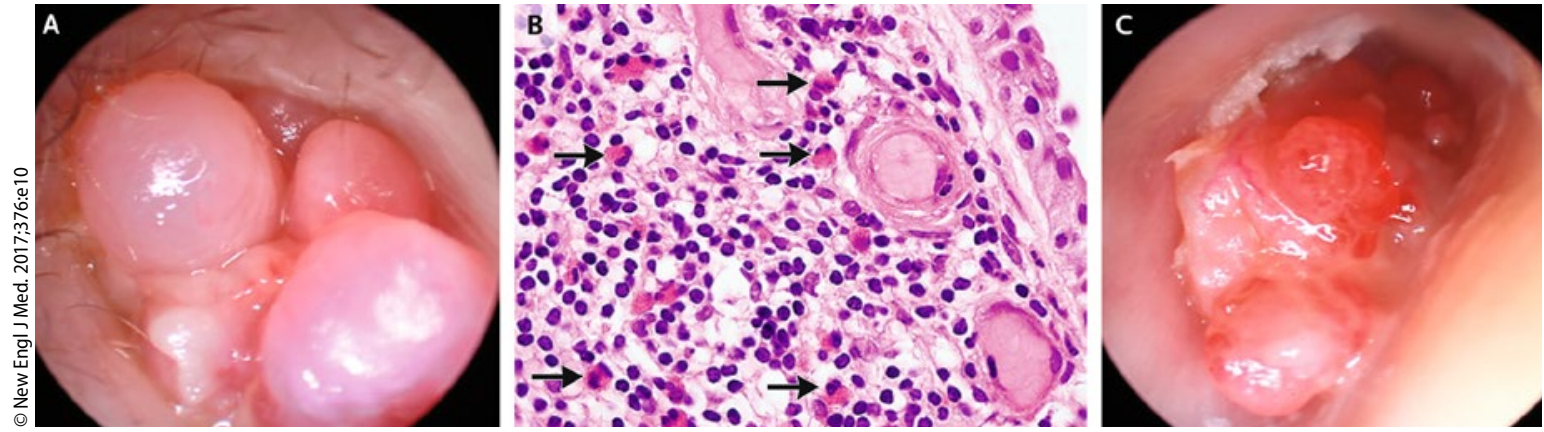

A: Polypoide Strukturen im rechten Ohr. B: Polypengewebe mit Eosinophilen (Pfeile). C: Rezidiv nach vier Monaten.

gemischter, vorwiegend aus Eosinophilen bestehender Infiltration (Abb. B). Auch im peripheren Blut bestand eine Eosinophilie mit

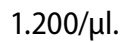

Man stellte die Diagnose einer eosinophilen Otitis media. Diese seltene, chronische und

Ein 50-jähriger Mann hatte seit einem halben Jahr beidseitig Ohrenschmerzen, intermittierendes Ohrenlaufen und zunehmenden Hörverlust. Aus der Anamnese waren ein Asthma bronchiale, Sinusitiden mit Nasenpolypen und eine Überempfindlichkeit gegen ASS bekannt. Bei der Otoskopie erkannte man polypoide Strukturen in beiden Gehörgängen, die aus dem Mittelohr durch das Trommelfell hervorragten und eine viskose Flüssigkeit absonderten (Abb. A). Die mikroskopische Untersuchung dieses Exsudats zeigte akute Entzündungszellen mit massenhaft Eosinophilen. In der Biopsie der Polypen fand sich ein Granulationsgewebe mit nur symptomatisch behandelbare Form erzeugt eine hoch viskose Otorrhö und geht häufig mit Nasenpolypen und Asthma einher. Der Patient wurde topisch und systemisch mit Steroiden behandelt, worunter sich Ohrenschmerzen und Otorrhö etwas besserten. Danach wurden die polypoiden Massen reseziert und eine Tympanoplastik vorgenommen. Im rechten Ohr wurde ein Hörgerät implantiert. Vier Monate später allerdings stellte sich der Patient mit einem Rezidiv in beiden Ohren vor (Abb. C).

Prof. Dr. med. H. S. Füeß|

- Lara-Sánchez H, Vellejo LA. Eosinophilic otitis media. New Engl J Med. 2017;376:e10 